\title{
Venous leg ulcers treated with fish collagen gel in a 12-week randomized single-centre study
}

\author{
Justyna Cwajda-Białasiki, ${ }^{1,2}$, Paulina Mościcka ${ }^{1,2}$, Maria T. Szewczyk ${ }^{1,2}$, Dorota Hojan-Jezierska ${ }^{3}$, \\ Weronika Kawałkiewicz', Anna Majewska3 ${ }^{3}$, Marta Janus-Kubiak ${ }^{4}$, Leszek Kubisz ${ }^{4}$, Arkadiusz Jawień ${ }^{5}$
}

${ }^{1}$ Department of Perioperative Nursing, Ludwik Rydygier Collegium Medicum in Bydgoszcz, Nicolaus Copernicus University in Torun, Poland

2Outpatient Department for Chronic Wound Management, University Hospital No. 1, Bydgoszcz, Poland

${ }^{3}$ Department of Hearing Healthcare Profession, Chair of Biophysics, Poznan University of Medical Science, Poznan, Poland

${ }^{4}$ Department of Biophysics, Chair of Biophysics, Poznan University of Medical Science, Poznan, Poland

${ }^{5}$ Department of Vascular Surgery and Angiology, Ludwik Rydygier Collegium Medicum in Bydgoszcz, Nicolaus Copernicus University in Torun, Poland

Adv Dermatol Allergol 2022; XXXIX (4): 714-722

DOI: https://doi.org/10.5114/ada.2021.108424

\begin{abstract}
Introduction: Venous ulcers are difficult to heal chronic wounds. Most challenging are the non-healing and recurrent ulcers, because of which we are seeking for therapies that stimulate and support their healing.

Aim: To assess the efficacy of fish collagen and its impact on the process of healing of venous leg ulcers.

Material and methods: This 12-week randomized single-centre study included a total of 97 adults with venous leg ulcer, randomized into two groups: the study group $(n=48)$ and the control group $(n=49)$. All patients received specialist medical care including skin and limb care, wound development according to the TIME scheme (tissue debridement, infection and inflammation control, moisture balance, edge) and short-stretch bandage compression (compression class II). In addition, the study group received tropocollagen gel applied to the skin around the wound. Every 2 weeks we evaluated the wound surface and temperature distribution on the shin (average temperature, median, minimum and maximum) - symmetrically on the sick and healthy limb.

Results: We observed a greater number of complete ulcer healing in the study group (week 12: 29.2\% vs. 22.4\%; week $24: 52.1 \%$ vs. $36.7 \%$ ). Faster healing time in the study group was observed in both big and small ulcers. The thermographic analysis showed statistically significant reduction in periwound inflammation among patients from the study group $(p<0.05)$. Statistically significant reduction in mean as well as maximum values of limb temperature occurred only in the study group (Tx: $R=0.37, p<0.001$; Tmax: $R=0.40, p<0.001$ ).

Conclusions: Thermographic analysis showed better wound healing effects in the group using tropocollagen. Significant impact of collagen on the healing process was observed only after 8 weeks of application.
\end{abstract}

Key words: venous leg ulcers, chronic wounds, fish collagen, planimetry, infrared thermography.

\section{Introduction}

Venous leg ulcers (VLUs) belong to the category of the most common chronic wounds of the lower limb. They constitute the most severe complication of chronic venous insufficiency (CVI). Approximately $2 \%$ of the adult population and $5 \%$ of senior citizens (over 65 years of age) develop active or healed ulceration [1-6]. The main etiological factors of VLUs are chronic venous hypertension and venous stasis, which is why compression therapy remains the mainstay of treatment for the majority of patients. Treatment outcomes depend on multiple prognostic factors, such as duration and wound size, recurrence rate and anatomical location of venous pathology [7-9]. The majority of ulcers are slow-healing wounds with a high recurrence rate of $70 \%$ and a $60 \%$ risk of becoming chronic $[10,11]$. Particularly troublesome are the so-called non-healing wounds, which will not heal for many years. Approximately $20 \%$ of ulcers are reported to remain active for about 2 years and $8-10 \%$ remain active for 5 years. Ulcers active for over 10 years have also been

Address for correspondence: Justyna Cwajda-Białasik, Department of Perioperative Nursing, Nicolaus Copernicus University, Ludwik Rydygier Collegium Medicum, 13-15 Jagiellońska St, 85-067 Bydgoszcz, Poland, e-mail: jcwajda@wp.pl, jcwajda-bialasik@cm.umk.pl Received: 26.04.2021, accepted: 9.06.2021. 
reported [3, 4, 6, 8, 9]. Chronic ulcers are characterized by permanent inflammation and high activity of proteolytic enzymes [12, 13]. Histological analyses among the patients with CVI have shown the infiltration of skin and subcutaneous tissue pericapillary space by multiple inflammatory cells, cytokines (interleukin $1 \alpha$ and $1 \beta-\mathrm{IL}-1 \alpha$ and IL-1 $\beta$, tumor necrosis factor $\alpha-$ TNF- $\alpha$ ), platelet adhesion activators, lysosomal proteolytic enzymes (mainly elastase), toxic oxygen metabolites and free radicals among others. In comparison with the control group, excessive catabolism of connective tissue and improper arrangement of collagen fibres have been observed among patients with VLUs [13-18]. In vitro studies on skin fibroblast culture have demonstrated that these pathologies may be the consequence of prolonged inflammation since typical inflammatory mediators decreased the synthesis of procollagen I and collagen and disrupted its development and structure. Similar observations have been conducted in fibroblasts cultured in fibrin gel [13, $19,20]$. Still, collagen is an essential structural protein, important in maintaining the mechanical function of the skin as well as wound healing. It constitutes more than $50 \%$ of all the proteins taking part in this process [13]. The exposure of collagen fibrils in damaged tissues sets in motion a chain of cellular and biochemical reactions which initiate haemostasis and subsequent proliferation. Collagen constitutes a mechanical framework for developing structures. Its synthesis, growth and arrangement have a vital influence on the final properties and resistance of scars. In addition to its scaffold function, collagen is a critical signalling molecule in the extracellular matrix [20]. The above-mentioned properties of collagen have long been used in the production of dressing materials, such as haemostatic dressings (collagen supports haemostasis) [21], biological dressings (in the treatment of chronic wounds and burns that are hard-toheal), bioactive dressings (collagen acts in synergy with other elements of the extracellular matrix, for instance, fibronectin, elastin, glycosaminoglycan) [13, 22-26] and antibacterial dressings (collagen utilized as a vehicle for an antibiotic to facilitate its penetration into deep structures of infected tissues) $[27,28]$. Collagen dressings usually do not produce side effects but their effects are limited to the wound bed. However, patients with VLUs also suffer from accompanying tissue trophic lesions (such as hemosiderosis, lipodermatosclerosis, atrophie blanche) and skin complications [5, 6, 29-32]. Many of them complain of persistent itch, excessive dryness and skin scaling. Some struggle with eczema - stasis dermatitis or contact (allergic) dermatitis, which increases the risk of microtrauma, infection and formation of new ulcers [20-34]. Intensive skin care, promoting its integrity and protective function may improve the effects of complex therapy of VLUs. Wounds start to heal from the edges, that is, the proliferation of granulation tissue and epidermis takes place as a result of the division of the surrounding healthy and intact cells. By contrast, the socalled aging cells show disrupted proliferative potential and they do not differentiate properly. That is why any intervention which aims to improve skin condition and promote healing should not be underestimated. We have assumed that topical application of collagen will reduce inflammation surrounding the wound and enable faster healing of ulcers. We used collagen in the form of gel intended to be applied directly on the skin around the wound, instead of the previously used dressing. So far fish collagen has been primarily used in the beauty industry as an ingredient in creams and skin care products in order to improve the overall condition of the skin on the face and body. To the best of our knowledge, no research into the influence of external use of fish collagen on the healing process of VLUs and the condition of periwound skin has been conducted.

\section{Aim}

The aim of this study was to assess the efficacy of fish skin collagen and its impact on the process of healing of VLUs. We assessed the progress of wound healing by planimetry and infrared thermography (IRT).

\section{Material and methods}

In this study we have exogenous collagen from the skin of freshwater fish. Collagen was obtained from farmed form of Silver carp skin (Collagen Active Science Sp. z o.o., Poznan, Poland). The manufacturer had controlled biological contamination of the material. It was found that the gel was free of microbiological contamination. Also all fatty acid was removed during the manufacturing process. No heavy metals were found. The collagen in the gel was in its native form, the denaturation temperature was found at $33.4^{\circ} \mathrm{C}$ (by viscometric method). The gel contained water, lactic acid, preservative (Rocoal $\mathrm{MD}$ ) and collagen of about $1 \%$ concentration. The electrophoretic studies showed the presence of $200 \mathrm{kDa}$ collagen $\beta$-chains and $130 \mathrm{kDa}$ collagen $\alpha$-chains. The gel was not applied directly to the wound and was a cosmetic product that met the standards for use on the skin of infants and around the eyes.

\section{Ethics statement}

The project received ethical clearance as a prerequisite of approval for funding from the National Centre of Research and Development as a part of Applied Research Projects (No. NCBiR, PBS3/B7/28/2015). The study protocol was approved by the local bioethics committee of the Nicolaus Copernicus University in Torun, and Ludwik Rydygier Collegium Medicum in Bydgoszcz (No. KB 69/2015) and conducted in accordance with the Helsinki Declaration of 1975. All enrolled patients signed a written 
informed consent form. Patients' data were managed in accordance with the Polish Data Protection Act [35].

\section{Participants}

This 12-week randomized single-centre study included 100 adults (> 18 years) with chronic venous ulcers. Recruitment occurred at a highly specialized national centre for chronic wound healing, in the period from 2016 to 2019. The study classification criteria included the presence of a leg ulcer (area between 5 and $50 \mathrm{~cm}^{2}$ ), CVI, as proven by scanning of the lower extremity blood vessels (duplex scan), a duration of ulceration > 3 months, an ankle brachial index (ABI) of 0.9-1.3, and a lack of clinical symptoms of infection. The exclusion criteria were ulcerations of mixed aetiology other than venous or undiagnosed and coexisting lower limb disorders. Eligible patients were randomized to either tropocollagen gel treatment (group A) or placebo alone (group B) using computer generated random numbers. Several patients withdrew during the study or missed follow-up visits after the wound healed. We included 97 patients in the final analysis ( $A, n=48 ; B, n=49$ ). Baseline characteristics of patients are shown in Table 1.

\section{Interventions}

All groups received standard wound care twice weekly for 12 weeks or until healing was complete. Standard treatment for VLUs included 2nd class compression therapy with short-stretch bandages Matopress and cotton wool pads Matosoft Natural (TZMO Matopat, Torun, Poland), used for skin protection purposes. Wound hygiene procedures [36] included cleansing the wound and periwound skin, debridement, renewing the wound edges, using the dressing appropriate to the healing phase. Moreover, patients in group A had tropocollagen gel applied on periwound skin, while patients in group B received placebo. We applied $5 \mathrm{~cm}^{3}$ of the formulation at a time (that is, 10 pumps of the dispenser). The gel was applied on clean skin within $2 \mathrm{~cm}$ from wound edges. The gel was massaged into the skin moistened with saline solution (approximately $10 \mathrm{ml}$ ) using circular finger motions (we wore disposable gloves). Halfway through the application process, we would again moisten the skin with saline ( $0.9 \%$ solution) and gently rub in the remaining gel that had not been absorbed fully. The total application time was equal to $15 \mathrm{~min}$. The application took place twice a day for 12 weeks (even if the wound had completely healed). The nurse applied the gel during appointments at the clinic, while patients applied it on their own at home. The patients had been instructed on how to take care of the wound earlier and each bottle of the gel came with a written instruction of the application process.

\section{Ulcer area}

We assessed the dynamics of wound healing by planimetry and infrared thermography every 2 weeks during a 12 -week study. Moreover, at week 24 from the beginning of the study the patients were invited to a single follow-up appointment, during which all the measurements were taken once again. Wound area calculations were performed using a Visitrak digital wound measuring device (Visitrak Digital: Digital-Pad, Smith \& Nephew, Austria). The primary endpoint, complete wound healing, was defined as a 100\% reduction in wound area. Secondary endpoints included reduction in wound area by $60 \%$, change in wound area (in $\mathrm{cm}^{2}$ and as a percent change from baseline), ulcer healing rate assessment for small $\left(<10 \mathrm{~cm}^{2}\right)$ and large $\left(\geq 10 \mathrm{~cm}^{2}\right)$ ulcers (in $\mathrm{cm}^{2}$ per week), and the percentage change (from baseline) in wound bed tissue composition, including epithelial tissue, granulation tissue, fibrin and sloughy tissue $[36,37]$.

Table 1. Baseline characteristics of the patients studied

\begin{tabular}{|c|c|c|c|c|}
\hline \multirow[t]{2}{*}{ Characteristic } & \multicolumn{2}{|c|}{ Group A $(n=48)$} & \multicolumn{2}{|c|}{ Group B $(n=49)$} \\
\hline & Mean \pm SD (range) & Median & Mean \pm SD (range) & Median \\
\hline Age [years] & $64.4 \pm 11.6(35-88)$ & 64.5 & $62.4 \pm 13.00(39-87)$ & 64 \\
\hline \multicolumn{5}{|l|}{ Gender*: } \\
\hline \multicolumn{5}{|l|}{ Female } \\
\hline \multicolumn{5}{|l|}{ Male } \\
\hline Duration of CVI [years] & $17.8 \pm 12.7(1-50)$ & 15 & $16.4 \pm 13.5(1-52)$ & 12.5 \\
\hline Duration of VLU [months] & $71.3 \pm 103.8(3-440)$ & 20 & $39.2 \pm 60.7(2-370)$ & 19 \\
\hline $\mathrm{BMI}$ & $30.7 \pm 7.3(19.4-58.9)$ & 30 & $30.7 \pm 6.6(20.1-58.9)$ & 30.5 \\
\hline Initial wound size $\left[\mathrm{cm}^{2}\right]$ & $18.1 \pm 14.8(5-50)$ & 11.3 & $15.1 \pm 14.2(5-50)$ & 8.7 \\
\hline $\mathrm{ABI}$ right & $1.09 \pm 0.11(0.88-1.33)$ & 1.06 & $1.13 \pm 0.14(0.8-1.5)$ & 1.15 \\
\hline$A B I$ left & $1.08 \pm 0.12(0.83-1.4)$ & 1.06 & $1.11 \pm 0.15(0.72-1.5)$ & 1.10 \\
\hline
\end{tabular}

*Shown as the number of observations (percentage). SD - standard deviation, CVI-chronic venous insufficiency, VLU - venous leg ulcer, ABI - ankle-brachial pressure index. 


\section{Infrared thermography (IRT)}

The severity of periwound inflammation was assessed by means of infrared thermography. We used mobile, high-resolution infrared camera (FLIR Systems, Wilsonville, OR, USA; model T650sc; $640 \times 480$ pixels). We took the photos in projection perpendicular to the anterior surface of the leg (the photo included the extremities from the knees to the feet). All photos were automatically saved in IRT and digital versions. Image analysis was conducted using FLIR Tools+ as software. The software automatically calculated the temperature in the area specified by the researcher. We analysed the mean, median and extreme values of temperature (minimum and maximum) on both lower extremities. According to the guidelines [38] for thermographic analysis, we compared the periwound temperature of the limb with an ulcer present to the contralateral area on the nonulcerated limb. This constituted a point of reference.

During the process of photo taking we followed the guidelines of the International Academy of Clinical Thermology (IACT) [38, 39]. We ensured optimal conditions in the research facility and prepared patients for examinations. After removing all clothes, bandages, and dressings from the legs, we placed the patient in the correct position for the photo to be taken. Acclimatization lasted at least $15 \mathrm{~min}$. If the wound was contaminated and required debridement, the acclimatization time was measured once the wound care procedures had ended. The collagen gel was applied after taking temperature. In this manner, we eliminated the direct influence of skin preparation and skin massage on the temperature of the extremities, which would have compromised important measurement data. The results of initial examination and detailed thermographic evaluation of the healing process in patients with VLUs were discussed in another publication [40].

Moreover, all patients were asked to write down their subjective perceptions such as pain, discomfort, drainage, burning sensation or other adverse reactions. Adverse reactions were classified as "present" or "absent"; their severity was assessed on a 5 -point scale $(1-$ absent, 2 - mild, 3 -moderate, 4 - quite severe, 5 - very severe).

\section{Results}

A greater number of complete ulcer healing was observed in the study group. By week 12 , there were minor differences between both groups (14 (29.2\%) vs. $11(22.4 \%))$. During week 24 , more pronounced differences were observed during the follow-up appointment (25 (52.1\%) vs. $18(36.7 \%))$. At that time, patients in the study group showed an increase in the number of ulcers healed by $15.4 \%$. The percentage of healed wound surface was greater in the study group but the difference became noticeable only in week 24 (Me: 100\% vs. 88.2\%). Wound healing progress was observed in both groups but the ulcers in the study group healed faster. Faster healing time in the study group was observed in both big as well as small ulcers. Big ulcers were characterized by a faster healing rate in weeks 6,12 and 24 of the observation. Statistically significant differences between groups occurred only in week 24 (Me: 0.19 vs. $0.10 \mathrm{~cm}^{2} /$ week; $p=0.005$ ). The healing rate of small ulcers until week 6 was comparable in both groups whereas during week 12 the healing rate increased in the study group, with the differences just at the limit of statistical significance (Me: 0.15 vs. $0.14 \mathrm{~cm}^{2} /$ week; $p<0.001$ ). By week 24 all small ulcers healed, both in the study group as well as the control group. We observed far greater growth in the epidermis, greater growth in the granulation tissue and decrease in the fibrin/necrosis in the wound in subsequent measurements in the study group. A detailed characteristics and healing process parameters in both groups were presented in Table 2 . Changes in the surface of wounds in the consecutive weeks of observation were shown in Figure 1.

The thermographic analysis showed statistically significant reduction in periwound inflammation among patients from the study group. We observed significant changes of all temperature values (that is, mean, median, maximum) from the periwound skin area with the exception of the minimum value. Mean and maximum temperature of the ulcerated limb decreased as time passed. Furthermore, in the study group the temperature of the ulcerated limb became equal to the temperature of the contralateral limb. Statistically significant reduction in mean as well as maximum values of limb temperature occurred only in the study group (Tx: $R=0.37$, $p<0.001$; Tmax: $R=0.40, p<0.001)$. Such relationship was not observed in the control group (Figure 2).

In both groups patients reported local reactions from the beginning of the observation. During the treatment, 2 patients from the study group and 1 patient from the control group complained of itch, rash, redness and skin maceration. These symptoms subsided when the dressing was changed to an absorbent one and an extra absorbent layer was used (2 patients). One patient required dermatological consultation. After 2 weeks of eliminating the allergen (most likely soap), the symptoms subsided. As for the skin symptoms reported at the beginning of the therapy, they subsided in $62.5 \%$ of patients from the study group and $35.8 \%$ of patients from the control group in the course of the study or once it ended (Table 3).

\section{Discussion}

This study shows that a 12-week treatment with fish collagen gel improves the healing of venous leg ulcers and the condition of periwound skin. Previous studies have confirmed the effectiveness of collagen dressing in the treatment of chronic wounds [13, 16, 19, 21-28]. The effectiveness of dressings containing porcine small intestine 
Justyna Cwajda-Białasik, Paulina Mościcka, Maria T. Szewczyk, Dorota Hojan-Jezierska, Weronika Kawałkiewicz, Anna Majewska, Marta Janus-Kubiak, Leszek Kubisz, Arkadiusz Jawień

Table 2. Wound healing parameters at selected time intervals

\begin{tabular}{|c|c|c|c|c|c|}
\hline Variable & Units & Group A & Group B & $P$-value & SMD \\
\hline \multicolumn{6}{|c|}{ The number (\%) of completely healed ulcers*: } \\
\hline & $n$ & 48 & 49 & & \\
\hline After 6 weeks & $n(\%)$ & $4(8.3)$ & $3(6.1)$ & 0.768 & 0.101 \\
\hline After 12 weeks & $n(\%)$ & $14(29.2)$ & $11(22.4)$ & 0.644 & 0.108 \\
\hline After 24 weeks & $n(\%)$ & $25(52.1)$ & $18(36.7)$ & 0.155 & 0.313 \\
\hline \multicolumn{6}{|c|}{ The number (\%) of ulcers healed in $60 \%^{*}$ : } \\
\hline & $n$ & 48 & 49 & & \\
\hline After 6 weeks & $n(\%)$ & $13(27.0)$ & $12(24.5)$ & 0.445 & 0.208 \\
\hline After 12 weeks & $n(\%)$ & $28(60.9)$ & $25(54.3)$ & 0.673 & 0.132 \\
\hline After 24 weeks & $n(\%)$ & $33(71.7)$ & $27(61.4)$ & 0.372 & 0.221 \\
\hline \multicolumn{6}{|c|}{ Healing rate - big ulcers $>10 \mathrm{~cm}^{2 \star *}$ : } \\
\hline & $n$ & 25 & 19 & & \\
\hline After 6 weeks & $\mathrm{cm}^{2} /$ week, Me (SD) & $1.08(0.86)$ & $0.81(1.49)$ & 0.467 & 0.217 \\
\hline After 12 weeks & $\mathrm{cm}^{2} /$ week, Me [IQR] & $0.75[0.02,1.18]$ & $0.44[-0.22,1.33]$ & 0.656 & 0.142 \\
\hline After 24 weeks & $\mathrm{cm}^{2} /$ week, Me $[\mathrm{IQR}]$ & $0.19[0.09,0.54]$ & $0.10[-0.07,0.65]$ & 0.542 & 0.005 \\
\hline \multicolumn{6}{|c|}{ Healing rate - small ulcers $>10 \mathrm{~cm}^{2 \star *}$ : } \\
\hline & $n$ & 23 & 30 & & \\
\hline After 6 weeks & $\mathrm{cm}^{2} /$ week, Me [IQR] & $0.75[0.57,0.92]$ & $0.65[0.43,0.90]$ & 0.285 & 0.354 \\
\hline After 12 weeks & $\mathrm{cm}^{2} /$ week, Me (SD) & $0.15(0.27)$ & $0.14(0.20)$ & 0.824 & 0.063 \\
\hline After 24 weeks & $\mathrm{cm}^{2} /$ week, Me [IQR] & $0.00[0.00,0.04]$ & $0.00[0.00,0.09]$ & 0.867 & 0.287 \\
\hline \multicolumn{6}{|c|}{ The median percent of the healed ulcer area*: } \\
\hline After 6 weeks & (\%) $\mathrm{Me}[\mathrm{IQR}]$ & $48.61[19.36,85.03]$ & $50.59[16.30,78.81]$ & 0.858 & 0.061 \\
\hline After 12 weeks & (\%) Me [IQR] & $70.43[38.43,100.00]$ & $67.79[33.55,98.15]$ & 0.606 & 0.135 \\
\hline After 24 weeks & (\%) $\mathrm{Me}[\mathrm{IQR}]$ & $100.00[53.56,100.00]$ & $88.23[45.22,100]$ & 0.235 & 0.278 \\
\hline
\end{tabular}

$S D$ - standard deviation, $p$-value ( ${ }^{*}$ Fisher's exact test, ${ }^{* *}$ nonparametric Kruskal-Wallis test), SMD - standardized mean difference, Me - median, IQR - interquartile range.

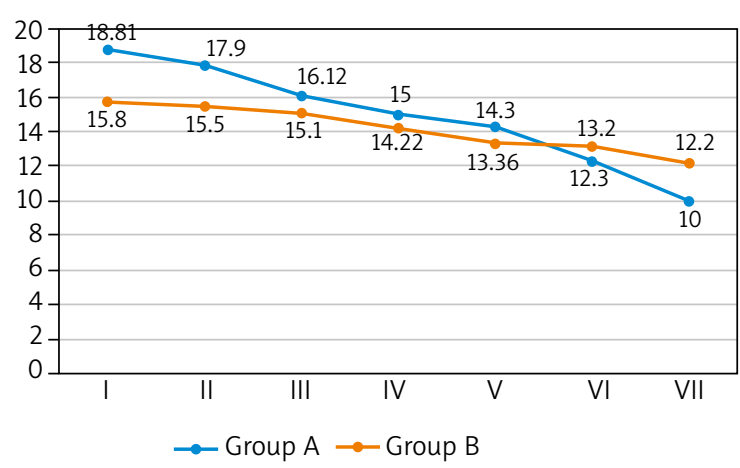

Figure 1. Comparison of ulcer healing indices in consecutive measurements in the studied groups

submucosa (SIS) [41-43] and bovine collagen in combination with oxidized regenerated cellulose (ORC) [44-46] has been well documented in randomized controlled trial (RCT). Such dressings were used in the treatment of chronic wounds of various aetiologies, including VLUs, mixed leg ulcers and DFUs. During the course of 12-week therapy,
$37-55 \%$ of wounds treated with collagen dressings healed, as compared to $28-34 \%$ of wounds treated in a standard manner. Good results were also obtained in several RCT assessing the acellular human dermal matrix [47, 48]. After 12-week therapy, healing was observed in 70-85\% of cases, as compared to $45-50 \%$ of DFUs; following 16 weeks, the results were $70 \%$ and $46 \%$, respectively. The results indicated the advantage of collagen but they were inconclusive. Diverse research methods, different clinical characteristics of wounds and small sample size made a reliable comparison impossible.

It is often stressed that some of the collagen production methods may be expensive and cost-ineffective, which results in the limited use of collagen biomaterials in the treatment of chronic wounds [13]. In our study we used collagen from the skin of freshwater fish (silver carp), which is inexpensive, easily available and characterized by good physicochemical properties [13, 24, 33, 34, 49]. Fish collagen has a relatively low molecular weight (less intermolecular crosslinking), which makes 
A

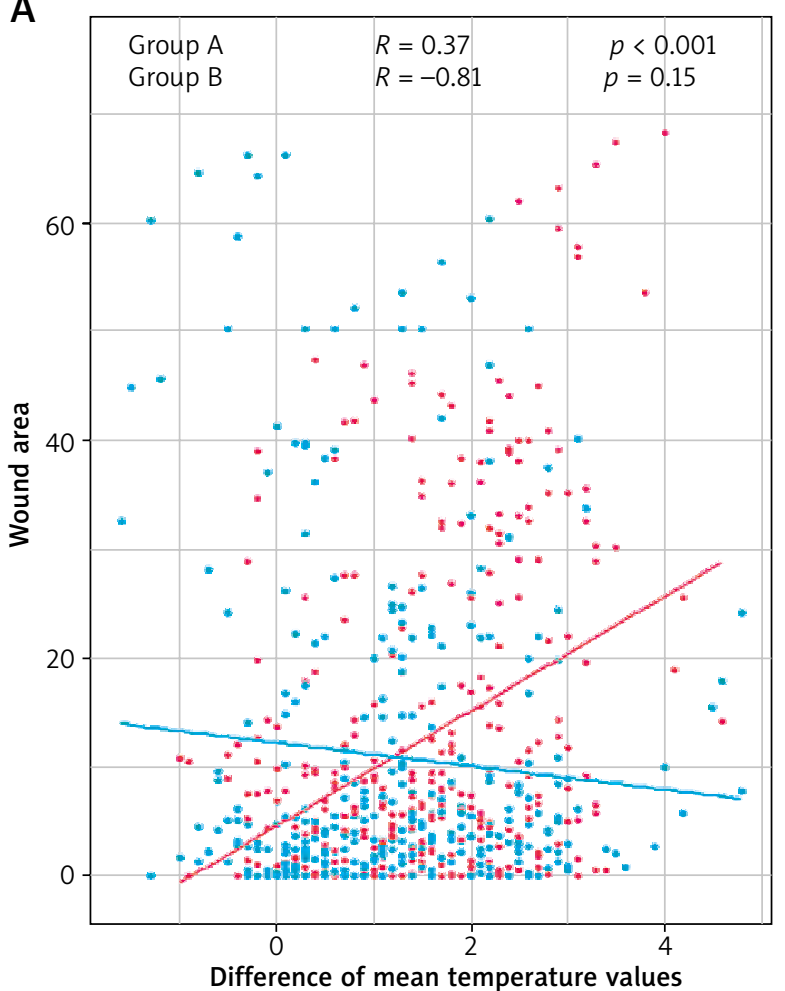

B

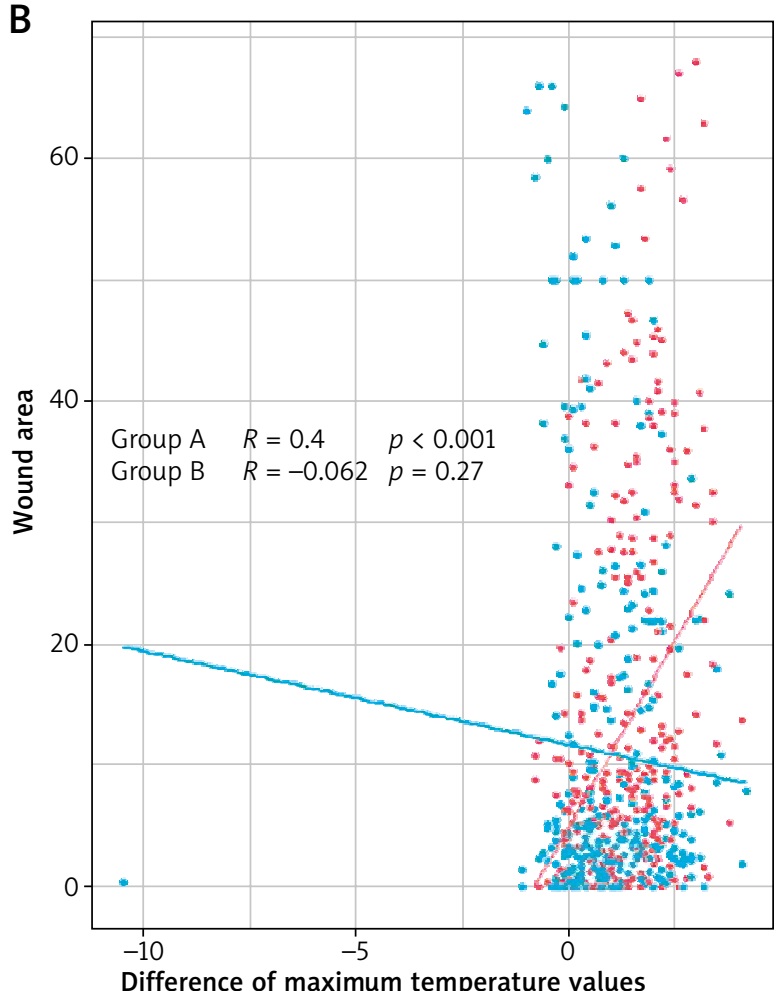

\begin{tabular}{lll}
$\mathbf{C}$ & & \\
\cline { 2 - 3 } Wound area & Group A & Group B \\
\hline Average temperature - ulcerated leg & 0.025 & -0.164 \\
\hline Maximum temperature - ulcerated leg & 0.046 & -0.128 \\
\hline Average temperature - non-ulcerated leg & -0.362 & -0.066 \\
\hline Maximum temperature - non-ulcerated leg & -0.370 & -0.060 \\
\hline Difference of mean values & 0.373 & -0.081 \\
\hline Difference of maximum values & 0.397 & -0.062 \\
\hline
\end{tabular}

Figure 2. Analysis of the dependence of average and maximum temperature values on changes in the wound area. A - Graph of correlation between the difference of mean temperatures and changes in the wound area. B - Graph of correlation between the difference of maximum temperatures and changes in the wound area. $\mathbf{C}$ - Characteristics of the correlation between temperature values and the healing process in the studied groups

it possible to obtain a fully biologically active molecule in the form of helix. Tropocollagen is highly biocompatible, nonallergenic and, as opposed to the land-based animal collagen, free from the risks of animal diseases and pathogens $[13,24]$. We conducted a pharmacoeconomic analysis which showed that the treatment with fish collagen is cheaper than the standard treatment of VLUs. The cost of obtaining one effect in the collagen treatment technology was 33\% lower than in the previous treatment technology [50].

We were the first to use fish collagen in the gel form on periwound skin, and not in the wound bed. In the pre- liminary analysis conducted in the group of 59 patients with VLUs we assessed the effect of the formulation on periwound skin and its ability to penetrate the corneal layer of the epidermis [49]. Electrical impedance measurements showed the improvement in the overall skin condition [49] while cutometry showed the improvement in skin elasticity and firmness after the use of collagen. In the placebo group, skin improvement was not significant $[49,51,52]$. It is worth noting that the positive effect of collagen on the skin was noticeable only from week 8 of the therapy [49]. This is also true in terms of the impact the collagen had on wound healing. By week 6 , the 
Table 3. Local skin symptoms/reactions

\begin{tabular}{lccccc}
\hline \multirow{2}{*}{ Variable } & \multirow{2}{*}{ Unit } & \multicolumn{2}{c}{ Group A } & \multicolumn{2}{c}{ Group B } \\
\cline { 3 - 6 } & & Before & After & Before & After \\
\hline Skin reactions* & & $24(50.0)$ & $9(18.7)$ & $28(57.1)$ & $18(36.7)$ \\
\hline Itch & $n(\%)$ & $11(22.9)$ & $2(4.2)$ & $12(24.5)$ & $5(10.2)$ \\
\hline Rash/allergy & $n(\%)$ & $5(10.4)$ & $1(2.1)$ & $4(8.2)$ & $2(4.1)$ \\
\hline Redness & $n(\%)$ & $9(18.7)$ & $3(6.3)$ & $11(22.4)$ & $714.3)$ \\
\hline Burning sensation & $n(\%)$ & $5(10.4)$ & $2(4.2)$ & $6(12.2)$ & $3(6.1)$ \\
\hline Skin maceration & $n(\%)$ & $6(12.5)$ & $3(6.3)$ & $4(8.2)$ & $3(6.1)$ \\
\hline Pain** & $n(\%)$ & $8(16.7)$ & $3(6.3)$ & $7(14.3)$ & $3(6.1)$ \\
\hline
\end{tabular}

*The number of patients with at least one skin reaction. ${ }^{* *}$ Pain not associated with the wound (periwound skin pain).

healing rate was similar in both groups and it was only between week 8-10 that the dynamics of ulcer healing in the study group improved. We obtained a greater number of complete healing (by $6.8 \%$ in week 12 and $15.4 \%$ in week 24), higher percentage of epidermal growth and granulation tissue as well as decrease in severity of subjective (such as pain, itch, burning sensation) and objective (redness, rash) skin symptoms. We may infer that the collagen gel applied on periwound skin first improved the overall skin condition and only secondarily enhanced the process of healing. In vitro studies have demonstrated that the topical use of collagen modulates chronic wound environment, mainly through the optimization of inflammation. The stabilization of the protease level and pro-inflammatory cytokines, whose abundance usually inhibits chronic wound healing [53-55], has been observed among others. We did not assess the physicochemical properties of the microenvironment of the wound and its surroundings, but compared the temperatures of the ulcerated limb to the contralateral area of the non-ulcerated limb. The difference of the maximum temperatures between an inflamed and a healthy area may equal approximately $1.5-2.2^{\circ} \mathrm{C}$ and even more in case of infection [56]. Reducing this difference is a good predictive factor, thus IRT assessment may prove helpful in the prognosis for wound healing $[40,56]$. It has been shown that higher temperature within the wound bed and its surroundings is associated with a several-times increased risk of delayed healing, and even wound deterioration. The authors have emphasized that the rise in wound temperature may be associated with critical colonization, infection and other factors which slow down healing. In this study, the temperature difference between the limbs decreased in the consecutive measurements. This value was significantly correlated with the progress of wound healing only in the study group. In the control group, the temperature differences did not decrease significantly and periwound inflammation persisted for a longer period of time.

An unquestioned strength of this study is the 12week follow-up period. To our knowledge, this is the first study to assess the progress of VLU treatment over such a long period of time using infrared thermography. Unfortunately, we have not assessed whether and for how long the effect on the skin and local inflammation persists after discontinuing the use of collagen. We believe that the use of collagen should be considered until the wound is healed.

\section{Conclusions}

The results described in this study suggest that the use of fish collagen in gel on the periwound skin is beneficial. Several weeks of therapy with the use of collagen have reduced inflammation and accompanying unpleasant sensations as well as improved healing of venous ulcers provided it is used in combination with standard wound bed debridement, hygiene and compression therapy of chronic venous insufficiency.

\section{Acknowledgments}

The authors would like to thank all of the patients and clinical staff for their cooperation in this study.

This study was financed by the National Centre of Development and Research as a part of Applied Research Projects (Grant NCBiR no. PBS3/B7/28/2015).

\section{Conflict of interest}

The authors declare no conflict of interest.

\section{References}

1. Mervis JS, Kirsner RS, Lev-Tov H. Protocol for a longitudinal cohort study: determination of risk factors for the development of first venous leg ulcer in people with chronic venous insufficiency, the VEINS (venous insufficiency in South Florida) cohort. BMJ Open 2019; 9: e023313.

2. Margolis DJ, Bilker W, Santanna J, Baumgarten M. Venous leg ulcer: incidence and prevalence in the elderly. J Am Acad Dermatol 2002; 46: 381-6.

3. Moffat CJ, Franks PJ, Doherty DC, et al. Prevalence of leg ulceration in a London population. QJM 2004; 97: 431-7. 
4. Nelzen O. Prevalence of venous leg ulcer: the importance of the data collection method. Phlebolymphology 2008; 15 : 143-50.

5. Evans CJ, Fowkes FGR, Ruckley CV, Lee AJ. Prevalence of varicose veins and chronic venous insufficiency in men and women in the general population: Edinburgh Vein Study. J Epidemiol Community Health 1999; 53: 149-53.

6. Jawień A, Grzela T, Ochwat A. Prevalence of chronic venous insufficiency (CVI) in men and women of Poland. Multicenter crosssectional study of 40095 patients. Phlebology 2003; 3: 110-22.

7. Reichard LE. Venous ulceration: compression as the mainstay therapy. J Wound Ostomy Continence Nurs 1999; 26: 39-47.

8. Szewczyk MT, Jawień A, Migdalski A, et al. Predicting time to healing by anatomical assessment of venous pathology. Med Sci Monitor 2009; 15: CR74-81.

9. Edwards HE, Parker CN, Miller C, et al. Predicting delayed healing: the diagnostic accuracy of a venous leg ulcer risk assessment tool. Int Wound J 2018; 15: 258-65.

10. Mościcka P, Szewczyk MT, Jawień A, et al. Subjective and objective assessment of patients' compression therapy skills as a predicator of ulcer recurrence. J Clin Nurs 2016; 25: 1969-76.

11. Skene AI, Smith JM, Dore CJ, et al. Venous leg ulcers: a prognostic index to predict time to healing. Br Med J 1992; 305: 1119-21.

12. Schultz GS, Wysocki A. Interactions between extracellular matrix and growth factors in wound healing. Wound Repair Regen 2009; 17: 153-62.

13. Gould LJ. Topical collagen-based biomaterials for chronic wounds: rationale and clinical application. Adv Wound Care 2016; 5: 19-31.

14. Leu AJ, Leu HJ, Franzeck UK, Bollinger A. Microvascular changes in chronic venous insufficiency - a review. Cardiovasc Surg 1995; 3: 237-45.

15. Jünger $M$, Steins A, Hahn M, Häfner HM. Microcirculatory dysfunction in chronic venous insufficiency (CVI). Microcirculation 2000; 7: S3-12.

16. Black E, Vibe-Petersen J, Jorgensen LN, et al. Decrease of collagen deposition in wound repair in type 1 diabetes independent of glycemic control. Arch Surg 2003; 138: 34-40.

17. Lerman OZ, Galiano RD, Armour M, et al. Cellular dysfunction in the diabetic fibroblast: impairment in migration, vascular endothelial growth factor production, and response to hypoxia. Am J Pathol 2003; 162: 303-12.

18. Cook H, Stephens P, Davies KJ, et al. Defective extracellular matrix reorganization by chronic wound fibroblasts is associated with alterations in TIMP-1, TIMP-2, and MMP-2 activity. J Invest Dermatol 2000; 115: 225-33.

19. Volk SW, Iqbal SA, Bayat A. Interactions of the extracellular matrix and progenitor cells in cutaneous wound healing. Adv Wound Care 2013; 2: 261-72.

20. Pastar I, Stojadinovic O, Yin NC, et al. Epithelialization in wound healing: a comprehensive review. Adv Wound Care 2014; 3: 445-64.

21. Lee $\mathrm{CH}$, Singla A, Lee Y. Biomedical applications of collagen. Int J Pharm 2001; 221: 1-22.

22. Friess W. Collagen: biomaterial for drug delivery. Eur J Pharm Biopharm 1998; 45: 113-36.

23. Rao KP. Recent developments of collagen-based materials for medical applications and drug delivery systems. J Biomater Sci Polym Ed 1995; 7: 623-45.
24. Jafari H, Lista A, Siekapen MM, et al. Fish collagen: extraction, characterization, and applications for biomaterials engineering. Polymers 2020; 12: 2230.

25. Oropallo AR. Use of native type I collagen matrix plus polyhexamethylene biguanide for chronic wound treatment. Plast Reconstr Surg Glob Open 2019; 7: e2047.

26. Bain MA, Thibodeaux KT, Speyrer MS, et al. Effect of native type I collagen with polyhexamethylene biguanide antimicrobial on wounds: interim registry results. Plast Reconstr Surg Glob Open 2019; 7: e2251.

27. Schimmer C, Ozkur M, Sinha B, et al. Gentamicin-collagen sponge reduces sternal wound complications after heart surgery: a controlled, prospectively randomized, doubleblind study. J Thorac Cardiovasc Surg 2011; 143: 194-200.

28. Stafiej JM, Szewczyk MT. Treatment of full-thickness pressure ulcers with a gentamicin sponge: a case report. J Wound Ostomy Continence Nurs 2012; 39: 331-41.

29. Rabe E, Berboth G, Pannier F. Epidemiology of chronic venous diseases. Wien Med Wochenschr 2016; 166: 260-3.

30. Jawień A, Szewczyk MT, Kędziora-Kornatowska K, et al. Functional and biopsychosocial restrictions among patients with a venous ulcer. Arch Med Sci 2006; 2: 36-41.

31. Szewczyk MT, Jawień A, Cwajda J. Skin integrity disorders among patients with chronic venous insufficiency and venous ulcer. Adv Dermatol Allergol 2005; 22: 141-7.

32. Herber OR, Schnepp W, Rieger MA. A systematic review on the impact of leg ulceration on patients' quality of life. Health Qual Life Outcomes 2007; 5: 44.

33. Gauza M, Kubisz L. Electrical conductivity of fish skin collagen in the temperature range 290-380 K. Acta Phys Polonica 2010; 118: 54-7.

34. Gauza-Włodarczyk M, Kubisz L, Mielcarek S, Włodarczyk D. Comparison of thermal properties of fish collagen and bovine collagen in the temperature range 298-670K. Mater Sci Eng C Mater Biol Appl 2017; 80: 468-71.

35. The Personal Data Protection Act of 10 May 2018 (Journal of Laws from 2018, item 1000).

36. Murphy C, Atkin L, Swanson T, et al. International consensus document. Defying hard-to-heal wounds with an early antibiofilm intervention strategy: wound hygiene. J Wound Care 2020; 29 (Suppl 3b): S1-28.

37. Schultz GS, Sibbald RG, Falanga V, et al. Wound bed preparation: a systematic approach to wound management. Wound Repair Regen 2003; 11 (1 Suppl): S1-28.

38. International Academy of Clinical Thermology Medical Infrared Imaging Standards and Guidelines. 2015. [online]. Available at file:///C:/Users/user/AppData/Local/Temp/20 15IACTStandardsandGuidelines-1.pdf (accessed April 2020).

39. Ammer K. The glamorgan protocol for recording and evaluation of thermal images of the human body. Thermol Int 2008; 18: 125-44.

40. Cwajda-Białasik J, Mościcka P, Jawień A, Szewczyk MT. Infrared thermography to prognose the venous leg ulcer healing process - preliminary results of a 12 -week, prospective observational study. Wound Repair Regen 2020; 28: 224-33.

41. Mostow EN, Haraway GD, Dalsing M, et al. Effectiveness of an extracellular matrix graft (OASIS Wound Matrix) in the treatment of chronic leg ulcers: a randomized clinical trial. J Vasc Surg 2005; 41: 837-43.

42. Niezgoda JA, Van Gils CC, Frykberg RG, Hodde JP. Randomized clinical trial comparing OASIS Wound Matrix to Regranex Gel for diabetic ulcers. Adv Skin Wound Care 2005; 18: 258-66. 
43. Romanelli M, Dini V, Bertone MS. Randomized comparison of OASIS wound matrix versus moist wound dressing in the treatment of difficult-to-heal wounds of mixed arterial/venous etiology. Adv Skin Wound Care 2010; 23: 34-8.

44. Veves A, Sheehan P, Pham HT. A randomized, controlled trial of Promogran (a collagen/oxidized regenerated cellulose dressing) vs standard treatment in the management of diabetic foot ulcers. Arch Surg 2002; 137: 822-7.

45. Gottrup F, Cullen BM, Karlsmark T, et al. Randomized controlled trial on collagen/oxidized regenerated cellulose/silver treatment. Wound Repair Regen 2013; 21: 216-25.

46. Vin F, Teot L, Meaume S. The healing properties of Promogran in venous leg ulcers. J Wound Care 2002; 11: 335-41.

47. Brigido SA. The use of an acellular dermal regenerative tissue matrix in the treatment of lower extremity wounds: a prospective 16-week pilot study. Int Wound J 2006; 3: 181-7.

48. Reyzelman A, Crews RT, Moore JC, et al. Clinical effectiveness of an acellular dermal regenerative tissue matrix compared to standard wound management in healing diabetic foot ulcers: a prospective, randomised, multicentre study. Int Wound J 2009; 6: 196-208.

49. Kubisz L, Hojan-Jezierska D, Szewczyk MT, et al. In vivo electrical impedance measurement in human skin assessment. Pure Appl Chem 2019; 91: 1481-91.

50. Wajszczuk K, Wawrzynowicz J, Sajna P, et al. An economic evaluation of a new medical technology of treatment of venous leg ulcers with fish collagen. Abstract. IV Symposium - Biophysics and Medicine; 2019 Sept 19-20; Poznań, Poland. Poznań: PUMS 2019.

51. Kawałkiewicz W, Janus-Kubiak M, Majewska A, et al. Evaluation of skin condition in the area of leg ulcer based on cutometry and results of the MPA program - case report. Abstract. IV Symposium - Biophysics and Medicine; 2019 Sept 19-20; Poznań, Poland. Poznań: PUMS 2019.

52. Majewska A, Janus-Kubiak M, Kawałkiewicz W, et al. Description of skin conditio in the area of the ulcer based on its electrical parameters - case report. Abstract. IV Symposium - Biophysics and Medicine; 2019 Sept 19-20; Poznań, Poland. Poznań: PUMS 2019.

53. Wiegand C, Abel M, Ruth P, et al. Effect of the sterilization method on the performance of collagen type I on chronic wound parameters in vitro. J Biomed Mater Res B Appl Biomater 2009; 90: 710-9.

54. Wiegand C, Schönfelder U, Abel M, et al. Protease and proinflammatory cytokine concentrations are elevated in chronic compared to acute wounds and can be modulated by collagen type I in vitro. Arch Dermatol Res 2010; 302: 419-28.

55. Chanmugam A, Langemo D, Thomason K, et al. Relative temperature maximum in wound infection and inflammation as compared with a control subject using long-wave infrared thermography. Adv Skin Wound Care 2017; 30: 406-41.

56. Nakagami G, Sanada H, lizaka S, et al. Predicting delayed pressure ulcer healing using thermography: a prospective cohort study. J Wound Care 2010; 19: 465-6, 468, 470. 